\title{
Orbit determination and control for the European Student Moon Orbiter ${ }^{\text {ts }}$
}

\author{
Federico Zuiani ${ }^{\mathrm{a}, *}$, Alison Gibbings ${ }^{\mathrm{a}}$, Massimo Vetrisano ${ }^{\mathrm{b}}$, Francesco Rizzi ${ }^{\mathrm{a}}$, Cesar Martinez ${ }^{\mathrm{a}}$, \\ Massimiliano Vasile ${ }^{\mathrm{b}}$
}

a Glasgow University, Space Advanced Research Team, Glasgow, United Kingdom

${ }^{\mathrm{b}}$ University of Strathclyde, Space Advanced Research Team, Glasgow, United Kingdom

\section{A R T I C L E I N F O}

\section{Article history:}

Received 4 March 2011

Received in revised form

30 September 2011

Accepted 2 March 2012

Available online 9 May 2012

\section{Keywords:}

ESMO

Weak Stability Boundary transfer

Navigation

Mission analysis

Flight dynamics

\begin{abstract}
A B S T R A C T
This paper presents the preliminary navigation and orbit determination analyses for the European Student Moon Orbiter. The severe constraint on the total mission $\Delta v$ and the all-day piggy-back launch requirement imposed by the limited available budget, led to the choice of using a low-energy transfer, more specifically a Weak Stability Boundary one, with a capture into an elliptic orbit around the Moon. A particular navigation strategy was devised to ensure capture and fulfil the requirement for the uncontrolled orbit stability at the Moon. This paper presents a simulation of the orbit determination process, based on an extended Kalman filter, and the navigation strategy applied to the baseline transfer of the 2011-2012 window. The navigation strategy optimally allocates multiple Trajectory Correction Manoeuvres to target a so-called capture corridor. The capture corridor is defined, at each point along the transfer, by back-propagating the set of perturbed states at the Moon that provides an acceptable lifetime of the lunar orbit. (c) 2012 Elsevier Ltd. All rights reserved.
\end{abstract}

\section{Introduction}

The considerable number of unmanned missions to the Moon, proposed or completed in the last decade, is the evidence of a revived interest in lunar exploration [1-3].

Scheduled for launch in 2014-15, the European Student Moon Orbiter (ESMO) [4,5] will be the first lunar

\footnotetext{
Abbreviations: ESMO, European Student Moon Orbiter; EKF, Extended Kalman Filter; GTO, Geostationary Transfer Orbit; NAC, Narrow Angle Camera; OD, Orbit Determination; STK, Satellite Tool Kit; TCM, Trajectory Correction Manoeuvres; WSB, Weak Stability Boundary; LEO, Low Earth Orbit; TLI, Trans Lunar Insertion

This paper was presented during the 61st IAC in Prague.

* Corresponding author. Tel.: +44 1415482083.

E-mail addresses: f.zuiani.1@research.gla.ac.uk (F. Zuiani), agibbing@eng.gla.ac.uk (A. Gibbings),

massimo.vetrisano@strath.ac.uk (M. Vetrisano),

francescorizzi86@hotmail.it (F. Rizzi),

0912682M@student.gla.ac.uk (C. Martinez),

massimiliano.vasile@strath.ac.uk (M. Vasile).
}

micro-satellite designed entirely by the student population and currently the only mission to the Moon planned by the European Space Agency for the near future. Using chemical propulsion, ESMO is devised to reach and enter a polar orbit, with a primary mission objective to acquire surface images of the South Pole. A Narrow Angle CCD Camera (NAC) will capture high resolution images over a period of six months. To complement the scientific return, the current optional secondary payloads include a small radar, a radiation monitoring experiment, a passive microwave radiometer, and a telecommunication experiment to test a lunar internet protocol.

In the recent past, a considerable number of mission studies analysed various options for transfers to the Moon: from simple Hohmann-like transfers [1] to ballistic transfers with phasing loops [6], from Low-Thrust spirals [7] to Weak Stability Boundary (WSB) transfers and other types of low-energy transfer via the L1 and L2 Lagrangian points [8].

In the case of ESMO, the limited available budget excludes a dedicated launch and imposes an all-day 
piggy-back payload launch requirement in which ESMO has little or no control over the launch date. This, coupled with the severe constraint on the $\Delta v$ budget, suggested the use of a WSB transfer after an extensive analysis of all other options [9]. A WSB transfer has the benefit of offering a higher degree of flexibility in the final selection of the launch vehicle and an associated reduction in $\Delta v$. However, this benefit comes with a higher sensitivity to the errors introduced by the launcher and the major $\Delta v$ manoeuvres. Therefore, a particular navigation strategy had to be devised to achieve the desired orbit around the Moon at minimum cost. The navigation strategy aims at maintaining the spacecraft within a capture corridor by optimally executing a number of TCM's throughout the transfer. The corridor is defined by the back propagation of the set of states, at the Moon, that ensures the required stability of the lunar orbit.

This paper presents the Orbit Determination (OD) analysis of ESMO and the proposed optimal trajectory control strategy to ensure capture at the Moon, for the baseline transfer scenario of the 2011-2012 launch window.

\section{Nominal transfer design}

The baseline solution at the end of the Phase A [10] envisaged a WSB-type of transfer from the Earth to the Moon. At the Moon the spacecraft would be inserted into a moderately elliptical polar orbit with orbital elements reported in Table 1.

The orbit defined in Table 1 provides repeated passages at low altitude over the South Pole of the Moon for at least 6 months. A WSB transfer, as illustrated in Fig. 1, was selected as it offers an inclination change and the raise of perigee at zero cost, therefore saving $\Delta v$.

In an ideal WSB transfer, the spacecraft needs to perform an initial Trans-lunar Insertion Manoeuvre (TLI) to be injected into the transfer trajectory to the Moon. The spacecraft then reaches a point above $10^{6} \mathrm{~km}$ from the Earth, in a wide region around the L1 or the L2 Lagrangian points of the Earth-Sun system. After performing a small (possibly zero) $\Delta v$ manoeuvre the spacecraft coasts back towards the Moon, where it is weakly captured. A final Lunar Orbit Insertion (LOI) manoeuvre ensures injection into the desired lunar orbit. In the case of ESMO each WSB transfer is designed by matching two separate legs: one from TLI to the weak stability region of the Earth-Sun system and one from the weak-stability region to LOI

Table 1

Parameters of the lunar orbit.

\begin{tabular}{ll}
\hline Parameter & Value \\
\hline$a$ & $3586 \mathrm{~km}$ \\
$e$ & 0.4874 \\
$i$ & $89.9^{\circ}$ \\
$\Omega$ & $63.8^{\circ}$ \\
$\omega$ & $292.9^{\circ}$ \\
$v$ & $0^{\circ}$
\end{tabular}

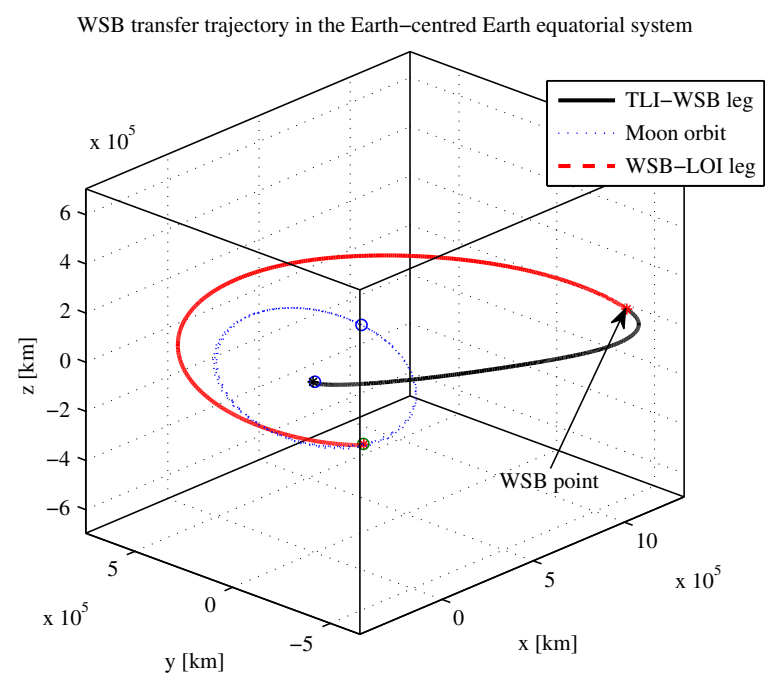

Fig. 1. WSB transfer trajectory in the Earth-centred equatorial reference frame.

[9,11]. The point at which the two legs are matched is here called WSB point and the corresponding manoeuvre, WSB manoeuvre.

From a computational point of view, a WSB transfer can be fully defined by the following 13 parameters:

- Departure date $t_{T L I}$.

- Duration of the TLI-WSB leg $t_{T L I-W S B}$.

- Duration of the WSB-LOI leg $t_{W S B-L O I}$.

- Modulus, azimuth and elevation of the $\Delta V$ of TLI manoeuvre defined in the Earth centred inertial reference frame $\Delta v_{T L I} \alpha_{T L I} \beta_{T L I}$.

- Modulus, azimuth and elevation of the $\Delta V$ of LOI manoeuvre defined in the Moon centred inertial reference frame $\Delta v_{L O I} \alpha_{L O I} \beta_{L O I}$.

- RAAN and true anomaly of the osculating Keplerian elements at departure, in the Earth-equatorial reference frame $\Omega_{G T O}, v_{G T O}$.

- RAAN and true anomaly of the osculating Keplerian elements at arrival, in the Moon-equatorial reference frame $\Omega_{L O}, v_{L O}$

The remaining Keplerian elements of the departure and arrival orbits are considered to be constant, since they are defined by the launcher insertion orbit and by the desired lunar orbit. The 13 scalar quantities define a design vector $\mathbf{y}=\left[t_{T L I} t_{T L I-W S B} t_{W S B-L O I} \Delta v_{T L I} \alpha_{T L I} \beta_{T L I} \Delta v_{L O I}\right.$ $\left.\alpha_{\text {LOI }} \beta_{\text {LOI }} \omega_{\text {GTO }} \Omega_{\text {GTO }} \omega_{\text {LO }} \Omega_{\text {LO }}\right]^{T}$.

The design vector $\mathbf{y}$ allows computing the Cartesian state vector of the spacecraft right after the TLI manoeuvre and before the LOI manoeuvre. Then, the orbital motion is propagated forwards in the TLI-WSB leg and backwards in the WSB-LOI leg. A gradient-based optimiser is then used to match the position of the two legs at WSB and to minimise the total $\Delta v$ of the transfer. The latter includes the cost of the TLI manoeuvre, the LOI manoeuvre and a WSB manoeuvre. The WSB manoeuvre is required to match the velocities of the two legs. For the propagation of the 
two legs, the motion of the spacecraft is governed by the following system of differential equations taking into account the gravitational attraction of the Earth, the Sun and the Moon:

$\dot{\mathbf{r}}=\mathbf{v}$

$\dot{\mathbf{v}}=-\frac{\mu_{E}}{r^{3}} \mathbf{r}-\mu_{S}\left(\frac{\mathbf{r}_{S S C}}{r_{S s c}{ }^{3}}-\frac{\mathbf{r}_{S E}}{r_{S E}{ }^{3}}\right)-\mu_{M}\left(\frac{\mathbf{r}_{M s c}}{r_{M s c}{ }^{3}}-\frac{\mathbf{r}_{M E}}{r_{M E}{ }^{3}}\right)$

where $\mathbf{r}$ and $\mathbf{v}$ are respectively the position and velocity vectors of the spacecraft with respect to the Earth in the J2000 inertial reference frame, $\mathbf{r}_{S S C}$ and $\mathbf{r}_{S E}$ are the Sunspacecraft and Sun-Earth vectors, $\mathbf{r}_{S S C}$ and $\mathbf{r}_{S E}$ are the Moon-spacecraft and Moon-Earth vectors, $\mu_{E}, \mu_{S}$ and $\mu_{M}$ are the planetary constants of Earth, Sun and Moon respectively. The position of Sun and Moon with respect to the Earth and the spacecraft are calculated using analytical ephemeris $[12,19]$ accounting for secular variations in the orbital elements of both the Earth and the Moon. An analytical model was used to describe the secular variation of the angles between the Earth-equatorial and the Moonequatorial reference frame [13]. The dynamic equations were numerically integrated with an explicit, variable step size, Runge-Kutta integration method with a $10^{-9}$ and $10^{-9}$ relative and absolute accuracies respectively.

In a nominal transfer, only the three manoeuvres mentioned above are required. However, given the sensitiveness of the transfer to even small variations in each of the manoeuvres and in the initial states, ESMO will require to perform a number of correction manoeuvres. The manoeuvres are planned and executed according to the estimation of the state vector of the spacecraft coming from a number of orbit determination campaigns.

\section{The capture corridor}

In order to successfully derive the accuracy requirement for orbit determination throughout the WSB transfer, leading to lunar insertion, the required level of accuracy at lunar insertion needs to be defined. An error in the determination of the exact lunar injection manoeuvre would directly translate into ESMO entering a deviated orbit around the Moon. This would imply a longer or shorter mission lifetime. Therefore, early analyses focused on investigating the influence of insertion errors (i.e. sensitivity) in the initial lunar orbital elements and their associated effect on the estimated orbital lifetime around the Moon. This early analysis permitted the definition of a capture corridor that guarantees the correct lunar insertion.

\subsection{Sensitivity analysis}

The sensitivity of ESMO's orbit around the Moon was assessed by randomly perturbing the nominal states at lunar insertion using a Latin Hypercube distribution [14]. The perturbation ranged from $1 \%$ to $5 \%$ of the nominal values of the orbital elements. For each error, ten sets of modified orbital elements were randomly generated. An example of perturbed orbital parameters is shown in Table 2.
Table 2

Lunar orbit: nominal parameters and error distribution for $1 \%$ error.

\begin{tabular}{llc}
\hline Parameter & Nominal value & $3 \sigma$ \\
\hline$a(\mathrm{~km})$ & 3586 & 35.86 \\
$e$ & 0.4874 & 0.0049 \\
$i$ (deg.) & 89.9 & 0.8990 \\
$\Omega$ (deg.) & 63.8 & 0.6380 \\
$\omega$ (deg.) & 292.9 & 2.929 \\
$v$ (deg.) & 0 & 0 \\
\hline
\end{tabular}

Table 3

Influence of insertion error against orbital decay time.

\begin{tabular}{lc}
\hline Error in orbital insertion (\%) & Decay time, $T+$ insertion (days) \\
\hline 1 & 159 \\
2 & 133 \\
3 & 119 \\
4 & 105 \\
5 & 81 \\
\hline
\end{tabular}

The orbital elements for each case were then propagated forward in time for six months or until ESMO crashed on the surface of the Moon. The AGI Satellite Tool Kit ${ }^{\circledR}$ (STK) [15] was used to propagate the orbit. The dynamic model included the inhomogeneous gravity field of the Moon with tesseral harmonics up to $21 \times 21$, plus the gravity pull of the Earth and the Sun. Because of the uncertainty on the value of the harmonic coefficients of the Moon, due to lack of observations on the far side, the propagation was repeated with different gravity models and for a variable number of harmonics [16], up to 60. For each case the temporal behaviour of the perilune altitude was assessed.

The increase in error corresponds to an increase in probability that ESMO would experience a reduction in orbital lifetime. Table 3 reports the average lifetime for a $1 \%$ error in orbital insertion. ESMO may experience a reduction in lifetime of approximately twenty days. For a $5 \%$ error instead, ESMO could experience a ninety-nine day reduction of its orbital lifetime. For the sake of the present analysis a reduction of up to 20 days was deemed to be acceptable to respond to the mission requirement that asks for an uncontrolled stable orbit for about six months [17]. Therefore, a $1 \%$ error was used throughout the remaining analyses.

Errors in the orbital elements can be translated into errors in the radial, transversal and out-of-plane components of position and velocity at the Moon. Figs. 2 and 3 show a displaced set of states for a $1 \%$ variation with respect to the nominal elements at the Moon. The $r-t-h$ reference frame is defined with respect to the Earth centred inertial reference frame at epoch. A zero variation corresponds to the nominal value. The level of acceptable error in the estimation of the states at the Moon will define the required tracking capability of the ground stations. Note that the goal of this analysis is only to derive an upper limit on the acceptable tracking error. 


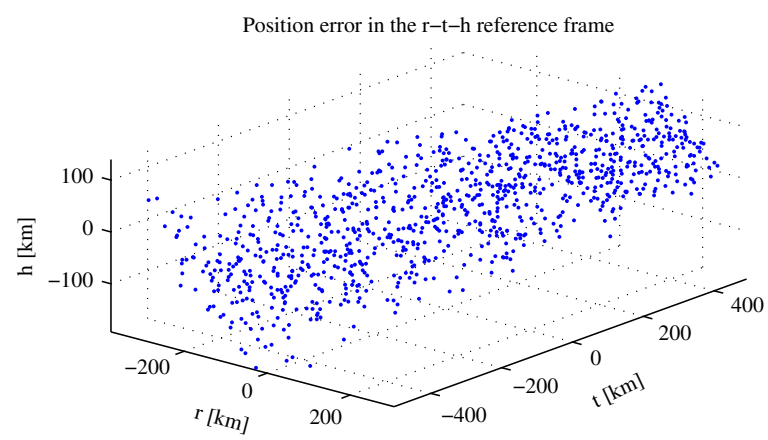

Fig. 2. Relative error in position projected along the radial, transversal and out-of-plane reference frame. The co-ordinate $(0,0,0)$ represents the nominal solution.

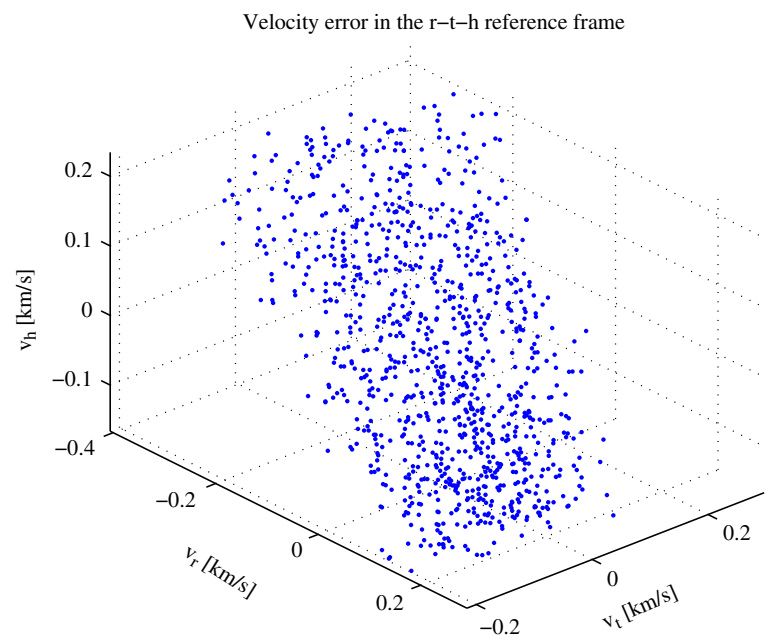

Fig. 3. Relative error velocity projected along the radial, transversal and out-of-plane reference frame. The co-ordinate $(0,0,0)$ represents the nominal solution.

\subsection{Corridor generation}

Assuming an error in the states at lunar injection derived from the $1 \%$ error in the orbital elements, a region of state space (position and velocity) at different times $\left(t_{\text {insertion }}-\Delta t\right)$ prior to the lunar orbital insertion was generated. This region, called the capture corridor, defines a set of positions and velocities that ESMO must have in order to be captured at the Moon at $t_{\text {insertion }}$ with the required accuracy.

The size of the capture corridor defines the required knowledge of ESMO's position and velocity along both legs of the WSB transfer. Orbit determination must therefore be able to discriminate with $99 \%$ probability $(3 \sigma)$ between whether or not ESMO is inside or outside the corridor at any time along the transfer. Without this level of accuracy, it would not be possible to predict whether or not ESMO is on course for lunar insertion. Note that inaccuracies or contingencies in the injection manoeuvre were not included within this analysis and will add up to the overall lifetime of the lunar orbit.
To assess the relative size of the corridor at $t_{\text {insertion }}$ $\Delta t$, the corridor must first be considered at $t_{\text {insertion. }}$ Fig. 4 defines the relative radial-transversal and out-of-plane reference frame at ESMO's nominal injection point, the radius $\mathbf{r}$ defines the position vector with respect to the Moon in the Moon equatorial reference frame. At the injection point, the insertion accuracy is given as a function of the error in position and velocity. This error is then propagated backwards. The set of backwards propagated states defines a region (or cloud) in the state space that surrounds the nominal solution. Each point inside the cloud represents a pair of position and velocity that will lead to capture at lunar insertion if the state is propagated forward.

The displacement $\delta \mathbf{r}$ and the velocity variation $\delta \mathbf{v}$ on the $r-h$ plane were randomly generated within a given range. The perturbed state vector $[\mathbf{r}+\delta \mathbf{r v}+\delta \mathbf{v}]$ was then propagated backwards for $\Delta t$. The displacement vector $\delta \mathbf{r}$ is defined as

$\delta \mathbf{r}=\delta r[\cos \theta, 0, \sin \theta]^{T}$

and the velocity variations $\delta \mathbf{v}$ are defined as follows:

$\delta \mathbf{v}=\delta v[\cos \vartheta \cos \phi, \cos \vartheta \sin \phi, \sin \vartheta]^{T}$

$\bar{\phi}=\frac{\phi}{2 \pi} ; \quad \bar{\vartheta}=\frac{\cos (\vartheta+\pi / 2)+1}{2}$

The quantities $\bar{\phi}$ and $\bar{\vartheta}$ are taken randomly within the interval $[0,1]$, with uniform distribution. The displacement norm $\delta r$ is taken from the interval $\left[0, \varepsilon_{r}\right]$, with uniform distribution, where $\varepsilon_{r}$ is the expected error in position. With this choice it is implicitly assumed that there is $100 \%$ probability that the displacement is in that interval. Therefore, there is a $100 \%$ probability that if ESMO is within the corridor at all times along the transfer, then it is captured at the Moon. The reverse is not true in general. For the velocities, the norm $\delta v$ is taken from the interval $\left[0, \varepsilon_{v}\right]$ with uniform distribution.

A total of 10,000 perturbed state vectors were propagated backwards from lunar injection to one week, two weeks along the transfer and up to the WSB point. The resulting positions and velocities were then projected onto the $r-h$ and $r-t$ planes at epoch. Fig. 5 shows a sketch of a perturbed solution intersecting the $r-h$ plane.

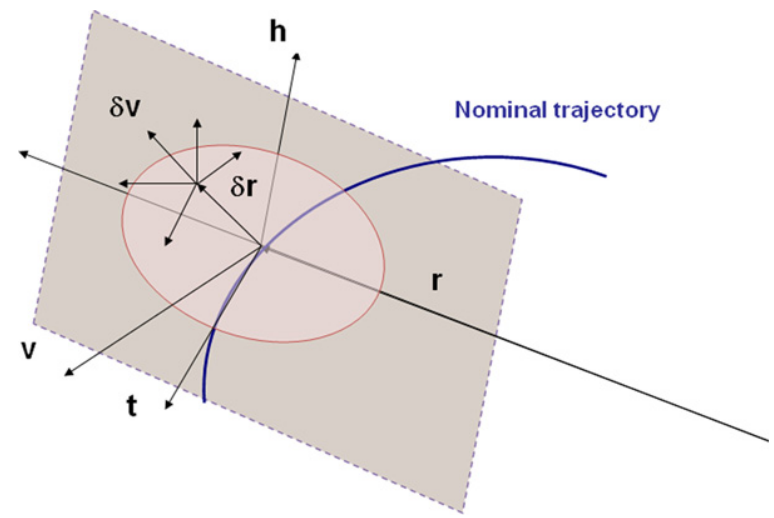

Fig. 4. Schematic of the $r-h$ plane at the lunar orbit insertion point. 


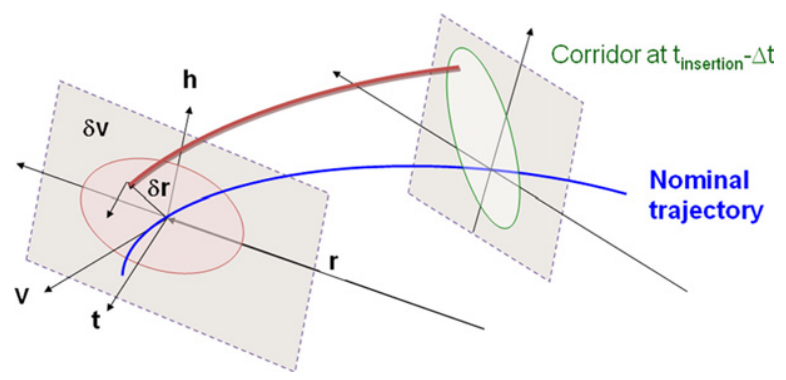

Fig. 5. Schematic of the backward propagation.

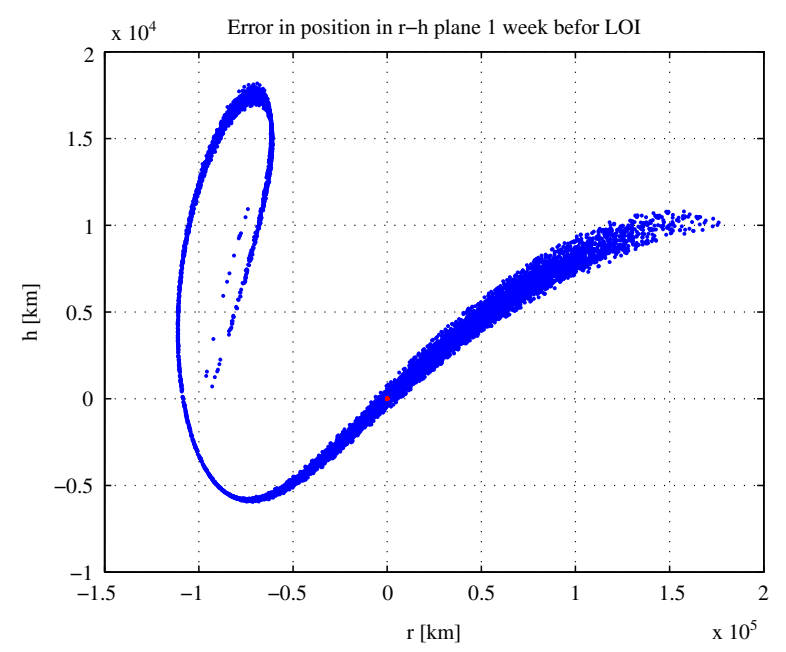

Fig. 6. Position dispersion at one week from lunar injection, $r-h$ plane.

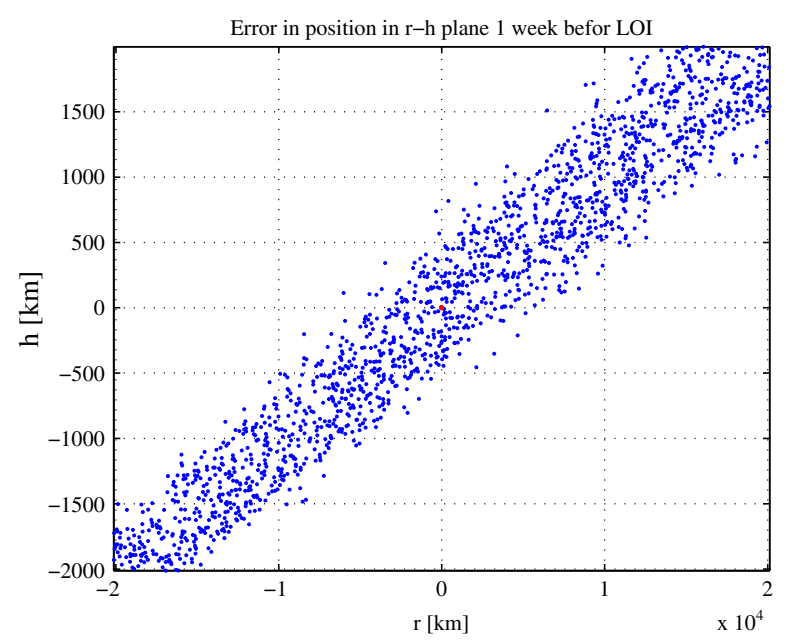

Fig. 7. Position dispersion at one week from lunar injection, $r-h$ plane (close up around the nominal transfer).

Figs. 6-8 show the results of the backward propagation (position and velocity dispersion in the $r-h$ plane) at one week from lunar injection for a trajectory departing on 16 March 2011. Errors used are $\varepsilon_{r}=5 \mathrm{~km}$ and $\varepsilon_{v}=10 \mathrm{~m} / \mathrm{s}$. Figs. 10-13 display the result of the propagation at two weeks before lunar injection. The position and velocity

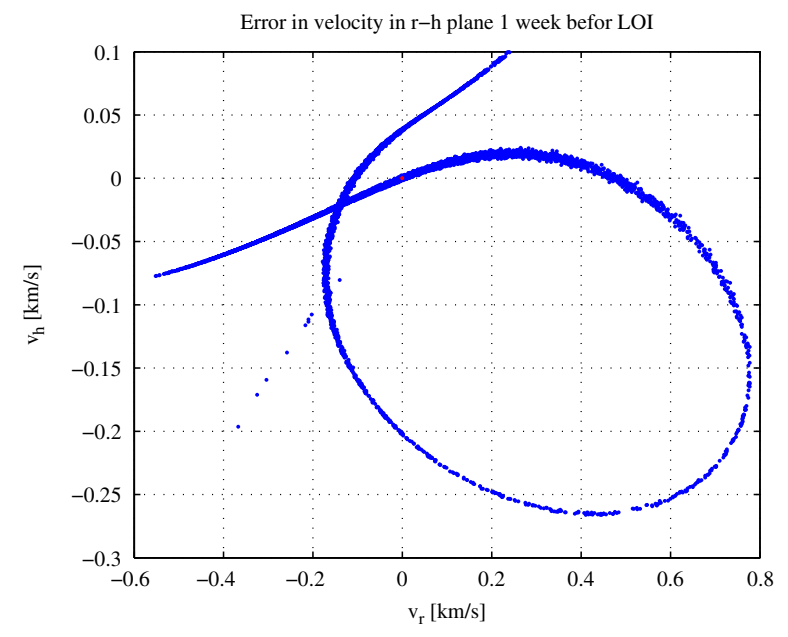

Fig. 8. Velocity dispersion at one week lunar injection, $r-h$ plane.

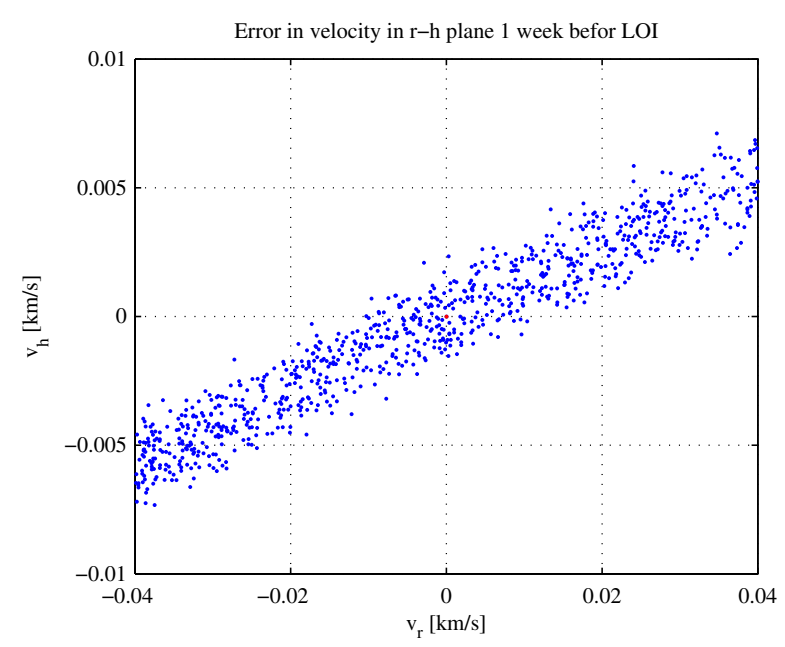

Fig. 9. Velocity dispersion at one week lunar injection, $r-h$ plane (close up around the nominal transfer).

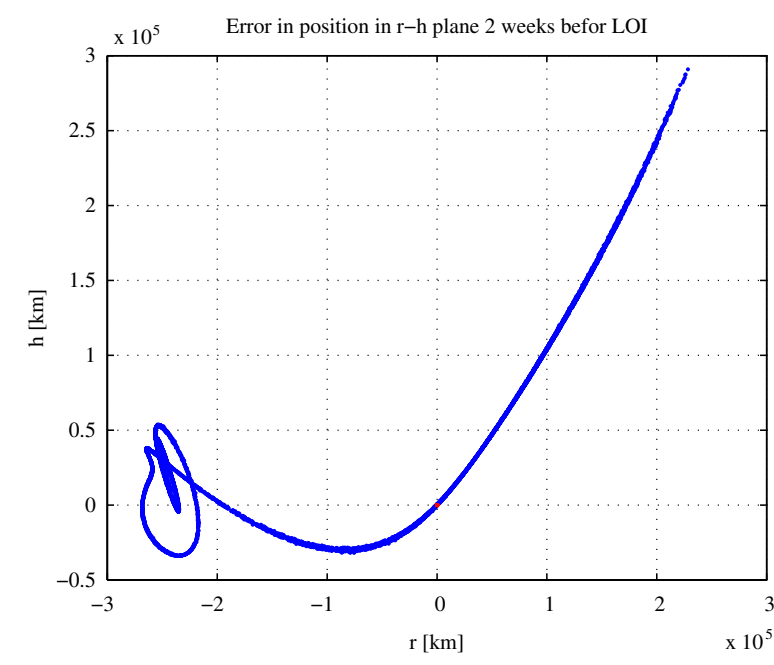

Fig. 10. Position dispersion at two weeks from lunar injection, $r-h$ plane. 


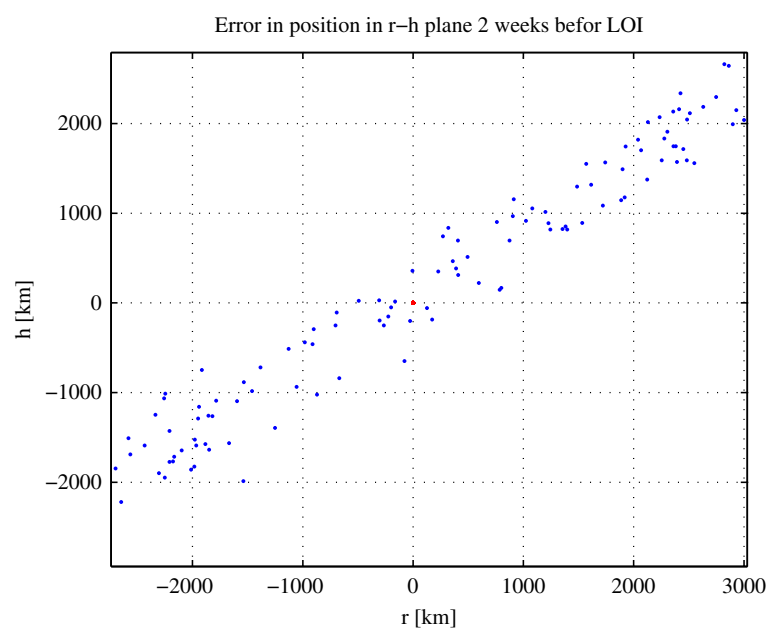

Fig. 11. Position dispersion at two weeks from lunar injection, $r-h$ plane (close up around the nominal transfer).

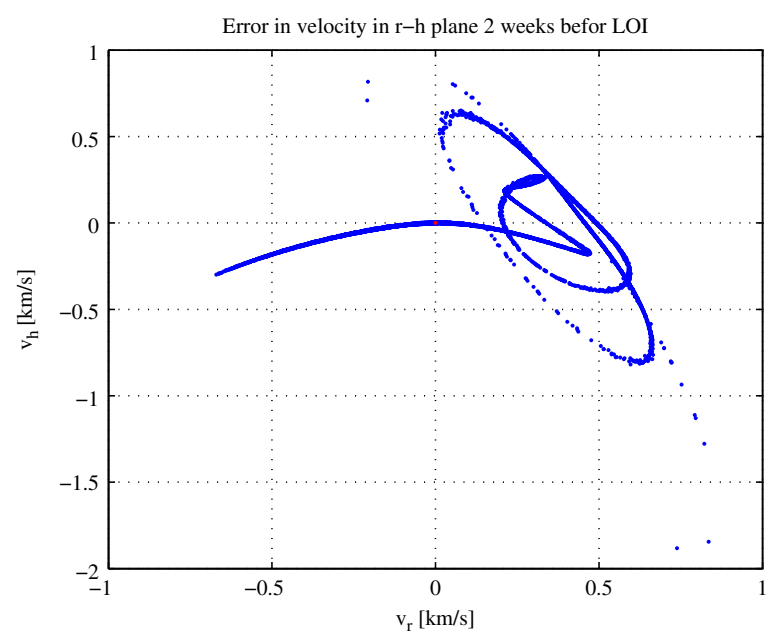

Fig. 12. Velocity dispersion at two weeks from lunar injection, $r-h$ plane.

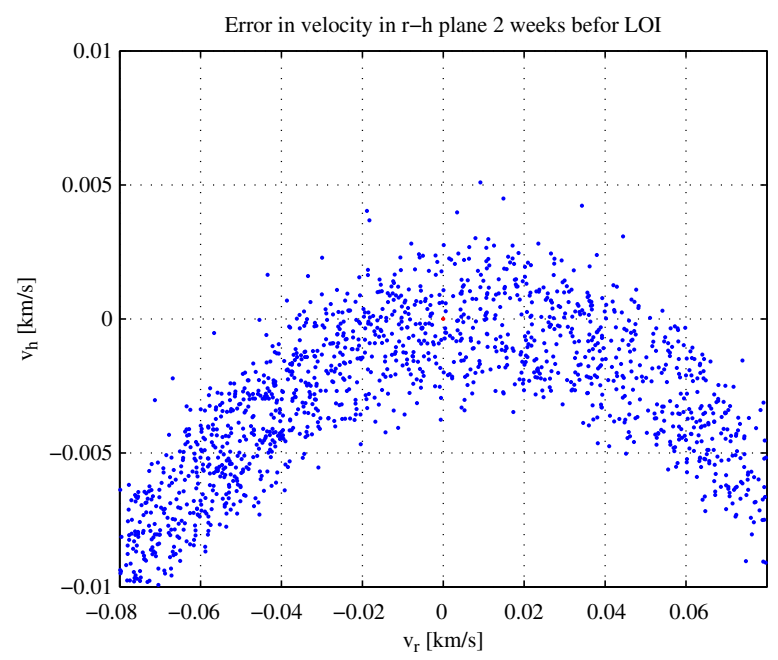

Fig. 13. Velocity dispersion at two weeks from lunar injection, $r-h$ plane (close up around the nominal transfer).
Table 4

Orbit determination accuracy requirements at 2 weeks from $t_{\text {injection. }}$.

\begin{tabular}{ll}
\hline Position & Velocity \\
\hline $25 \mathrm{~km}$ Radial (range) & $0.005 \mathrm{~km} / \mathrm{s}$ Radial (range rate) \\
$10 \mathrm{~km}$ Along track & $0.001 \mathrm{~km} / \mathrm{s}$ Along track \\
$10 \mathrm{~km}$ Out of plane & $0.001 \mathrm{~km} / \mathrm{s}$ Out of plane \\
\hline
\end{tabular}

plots report the variation with respect to the nominal value and, therefore, are centred around the origin. As long as ESMO is located within the trajectory corridor then orbital insertion around the Moon can be achieved.

Note that the trajectories corresponding to the curl (Figs. 6, 8, 10 and 12) will not reach the WSB region and do not represent feasible transfers. Furthermore, it is important to underline how the corridor tends to get thinner in the normal and transversal directions while it seems to stretch along the radial direction. Close ups in Figs. 7, 9, 11 and 13 allow for an estimation of the size of the corridor along the $r-h$ directions. From Fig. 7 one can see that, at one week, the corridor has a half-width of roughly $300 \mathrm{~km}$ along the radial and $400 \mathrm{~km}$ along the normal direction; in terms of velocity (Fig. 9), the dimensions are $0.01 \mathrm{~km} / \mathrm{s}$ along the radial and $0.001 \mathrm{~km} / \mathrm{s}$ along the out of plane directions. At two weeks before LOI, the half-width of the corridor in position (Fig. 11) is roughly $400 \mathrm{~km}$ along the radial and $300 \mathrm{~km}$ along normal directions; in velocity (Fig. 13), they are $0.03 \mathrm{~km} / \mathrm{s}$ and $0.002 \mathrm{~km} / \mathrm{s}$ respectively. The required orbit determination accuracy was derived from the size of the corridor at the farthest point from the Earth, along the transfer trajectory. Table 4 reports the worst-case required orbit determination accuracy at the WSB point assuming a 100\% margin, i.e. the maximum allowable error in position and velocity was reduced to less than half of the actual size of the corridor at the farthest point from the Earth.

\section{Orbit determination process}

The actual satisfaction of the OD requirements derived in the previous section was assessed by modelling and simulating the OD process with the tracking stations allocated to ESMO. As shown in Fig. 14, the output of the OD process was then used to allocate a number of trajectory correction manoeuvres in order to remain within the capture corridor at one week from lunar orbit insertion.

The diagram in Fig. 14 represents the algorithm simulating the orbit determination process and the optimal allocation of TCM's. The Orbit Determination processes range and range rate, angle and angles rate measurements, coming from a single ground station, and produces an estimate of the state of the spacecraft using a Kalman filter. State estimation is then used in the Navigation block (see Fig. 14), which generates an optimised sequence of correction manoeuvres.

The estimation accuracy, together with the additional errors introduced during the execution of the correction manoeuvres, has considerable effects on the Navigation 
budget and on the possibility for ESMO to be captured around the Moon. Therefore, the OD process is simulated by introducing random errors in the initial states of the nominal trajectory and in the measurements. The magnitude of the errors in the initial state was derived from the $3 \sigma$ launch dispersion: $1 \mathrm{~m} / \mathrm{s}$ in velocity and $1 \mathrm{~km}$ in position. The errors in the measurements instead were derived from the available information on the tracking stations. The set of measurements includes range and range rate $\rho$ and $\dot{\rho}$, from the ground station, plus the pointing angles and their variations with time $A, E, \dot{A}$ and $\dot{E}$ (respectively azimuth, elevation, azimuth rate and elevation rate). Since the actual position of the spacecraft is given in the Earth Centred Inertial (ECI) reference frame, it is necessary to write the state of ESMO as it was seen in the local South East Zenith (SEZ) reference frame of the ground station as shown in Fig. 15.

The range in the ECI reference frame is given by the difference in position of the spacecraft and ground station location:

$\boldsymbol{\rho}_{E C I}=\mathbf{r}_{E C I}-\mathbf{r}_{\text {site-ECI }}$

where $\mathbf{r}_{\text {site-ECI }}$ is the ECI position of the ground station. The range and velocity vectors in the SEZ frame are given by the following transformations:

$\boldsymbol{\rho}_{S E Z}=\mathbf{A}_{E C I-E C E F} \mathbf{A}_{E C I-E C E F}^{T} \boldsymbol{\rho}_{E C I}$

$\dot{\boldsymbol{\rho}}_{S E Z}=\mathbf{A}_{S E Z-E C E F} \mathbf{A}_{E C I-E C E F}^{T} \mathbf{v}_{E C I}$

where $\mathbf{A}_{S E Z-E C E F}$ and $\mathbf{A}_{E C I-E C E F}$ are respectively the transformation matrix from the Earth Centred Earth Fixed (ECEF) reference frame to SEZ and the transformation matrix from ECEF to ECI [20]:

$\mathbf{A}_{E C I-E C E F}=\left(\begin{array}{ccc}\cos \theta & -\sin \theta & 0 \\ \sin \theta & \cos \theta & 0 \\ 0 & 0 & 1\end{array}\right)$

$\mathbf{A}_{S E Z-E C E F}=\left(\begin{array}{ccc}\sin \phi \cos \lambda & -\sin \phi \sin & -\cos \\ -\sin \lambda & \cos \lambda & 0 \\ \cos \phi \cos & \cos \phi \sin & \sin \phi\end{array}\right)$

$\Phi$ and $\lambda$ are the latitude and longitude of the ground station; $\theta$ is the rotation angle between the ECI and ECEF reference frame about the $z$-axis:

$\theta=280.4606+\Omega t$

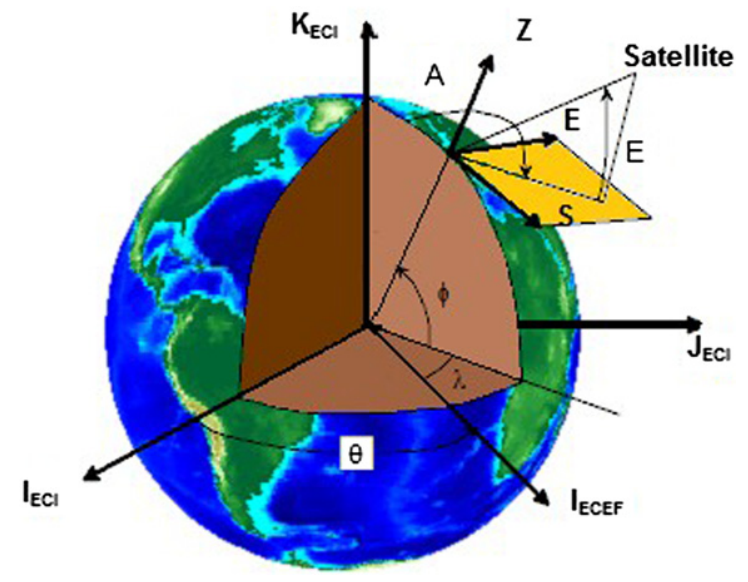

Fig. 15. Reference frames [20].

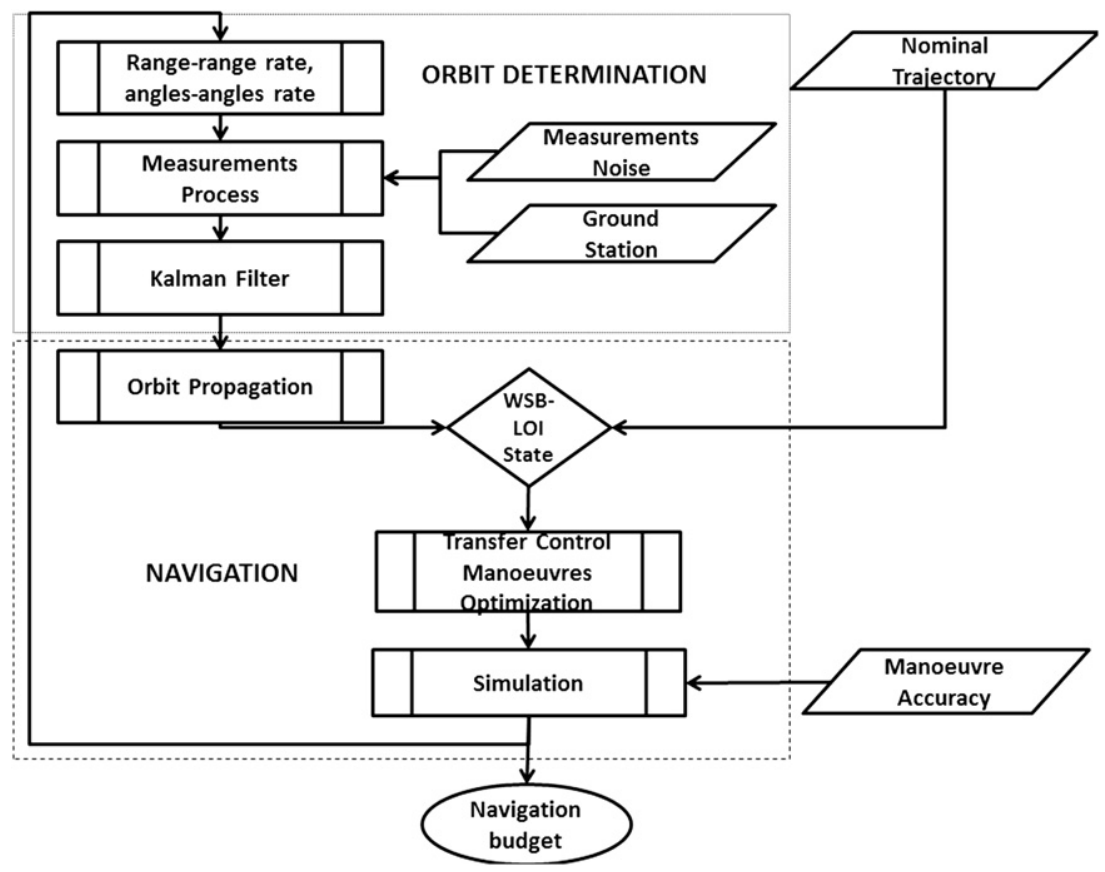

Fig. 14. Orbit determination and navigation algorithm. 
where $\Omega$ is the Earth's angular speed expressed in deg/day and $t$ is the time expressed in MJD2000. Finally the set of simulated measurements is obtained from the SEZ position and velocity:

$\rho=\left|\boldsymbol{\rho}_{\text {SEZ }}\right|$

$E=\operatorname{asin} \frac{\rho_{Z}}{\rho}$

$A=\operatorname{asin} \frac{\rho_{E}}{\sqrt{\rho_{S}^{2}+\rho_{E}^{2}}}$

$\dot{\rho}=\frac{\dot{\boldsymbol{\rho}}_{S E Z} \cdot \boldsymbol{\rho}_{S E Z}}{\rho}$

$\dot{A}=\frac{\dot{\rho}_{S} \rho_{E}+\dot{\rho}_{E} \rho_{S}}{\rho_{S}^{2}+\rho_{E}^{2}}$

$\dot{E}=\frac{\dot{\rho}_{Z}-\rho \sin E}{\rho_{S}^{2}+\rho_{E}^{2}}$

The actual measurements were simulated by perturbing the nominal ones with a random noise with normal distribution. Ionospheric and tropospheric refractions were not taken into account since their effect is expected to be corrected at ground station level before the information is provided to flight dynamics for orbit determination. The measurements are then processed by means of an Extended Kalman Filter [18,21] (EKF). This is a wellknown dynamic optimal filter which was first employed in the Apollo program. This kind of filter is suitable for both real-time and off-line applications. Within the ESMO programme the use of a sequential estimator has two motivations: since an autonomy experiment was proposed, the use of a sequential estimator, would allow both an efficient processing of the measurements on ground, with an online response to any contingent situation, and the testing of the autonomous navigation strategies; besides being a unique educational opportunity, ESMO offers the chance to experiment new solutions and working methodologies that can be used in future extremely low cost missions. The EKF assumes that the true state $\mathbf{x}=[\mathbf{r}, \mathbf{v}]^{\mathbf{T}}$ in the ECI reference frame is close to the estimated state. The dynamic and measurement model used in the filtering take the following form:

$\dot{\mathbf{x}}(t)=\mathbf{f}(\mathbf{x}(t), t)$

$$
\mathbf{y}_{k}=\mathbf{h}\left(\mathbf{x}_{k}\right)+\mathbf{v}_{k}
$$

where $\mathbf{f}(\mathbf{x}(t), t)$ is the set of nonlinear continuous-time equations represented in Eq. (1), $\mathbf{h}\left(\mathbf{x}_{k}\right)$ is the set of nonlinear discrete time equations for the measurements, coming from Eq. (6), and $v_{k}$ is the measurements noise defined as $\boldsymbol{v}_{k}=\mathbf{R} \boldsymbol{\sigma}_{n}$, where $\mathbf{R}$ is a diagonal matrix whose components along the diagonal are the squared values of the Ground Station errors reported in Table 5 (in the same order) and $\boldsymbol{\sigma}_{n} \in N(0,1)$ is a vector of random numbers taken from a normal distribution. Note that a minimal process noise was considered during the analysis because the dynamical model is expected to be fairly complete with the unmodeled components orders of magnitude lower than the modelled ones (e.g. solar pressure, inhomogeneous gravity field of the Earth, etc.). The system is characterised by an initial state estimate $\mathbf{x}_{0}$ and state covariance matrix $\mathbf{P}_{\mathbf{0}}$. The EKF is composed of two conceptually distinct phases: the time
Table 5

Magnitude of the random errors on measurements for OD simulation.

\begin{tabular}{ll}
\hline Measurement error & $3 \sigma$ \\
\hline$\Delta \rho$ & $30 \mathrm{~m}$ \\
$\Delta \dot{\rho}$ & $15 \mathrm{~cm} / \mathrm{s}$ \\
$\Delta \alpha$ & $10 \mathrm{Arcsec}$ \\
$\Delta \dot{\alpha}$ & $10^{-7} \mathrm{deg} / \mathrm{s}$ \\
$\Delta \beta$ & $10 \mathrm{Arcsec}$ \\
$\Delta \dot{\beta}$ & $10^{-7} \mathrm{deg} / \mathrm{s}$ \\
\hline
\end{tabular}

Table 6

Position and velocity errors at the end of the OD process.

\begin{tabular}{ll}
\hline OD duration & 3 days \\
\hline Difference in position $\overline{\Delta r}$ & $0.720 \mathrm{~km}$ \\
Difference in velocity $\overline{\Delta v}$ & $0.0106 \mathrm{~m} / \mathrm{s}$ \\
$\rho(\Delta r)$ & $0.4139 \mathrm{~km}$ \\
$\rho(\Delta v)$ & $0.0054 \mathrm{~m} / \mathrm{s}$ \\
\hline
\end{tabular}

update and the measurements update. The time update phase consists of the propagation of the latest estimate $\mathbf{x}_{k}^{+}$to obtain an a-priori estimate at current epoch $\mathbf{x}_{k+1}^{-}$ with the corresponding covariance matrix $\mathbf{P}_{k+1}^{-}$:

$\mathbf{x}_{k+1}^{-}=\mathbf{x}\left(t_{k+1} ; \mathbf{x}\left(t_{k}\right)=\mathbf{x}_{k}^{-}\right)$
$\mathbf{P}_{k+1}^{-}=\boldsymbol{\Phi}_{k+1} \mathbf{P}_{k}^{+} \boldsymbol{\Phi}_{k+1}^{T}$

where $\boldsymbol{\Phi}_{k+1}$ is the state transition matrix coming from the linearization of the dynamic equations about the updated state $\mathbf{x}_{k+1}^{-}$:

$\boldsymbol{\Phi}_{k+1}=\boldsymbol{\Phi}_{k+1}\left(t_{k+1}, t_{k}\right)=\mathbf{I}+\mathbf{F}_{k} \Delta t$

$\mathbf{F}_{k}=\frac{\partial \mathbf{f}\left(\mathbf{x}_{k}^{-}\right)}{\partial \mathbf{x}_{k}^{-}}$

The measurement update phase consists of the computation of the Kalman gain $\mathbf{K}_{k+1}$ and the state estimate $\mathbf{x}_{k+1}^{+}$and covariance matrix, $\mathbf{P}_{k+1}^{+}$updates

$$
\begin{aligned}
\mathbf{K}_{k+1} & =\mathbf{P}_{k+1}^{-} \mathbf{H}_{k+1}^{T}\left[\mathbf{H}_{k+1} \mathbf{P}_{k}^{-} \mathbf{H}_{k}^{T}+\mathbf{R}\right]^{-1} \\
\mathbf{x}_{k+1}^{+} & =\mathbf{x}_{k+1}^{-}+\mathbf{K}_{k+1}\left(\mathbf{y}_{k+1}-\mathbf{h}\left(\mathbf{x}_{k+1}^{-}\right)\right) \\
\mathbf{H}_{k+1} & =\frac{\partial \mathbf{h}\left(\mathbf{x}_{k+1}^{-}\right)}{\partial \mathbf{x}_{k+1}^{-}} \\
\mathbf{P}_{k+1}^{+} & =\left(\mathbf{I}-\mathbf{K}_{k+1} \mathbf{H}_{k+1}\right) \mathbf{P}_{k+1}^{-}
\end{aligned}
$$

where $\mathbf{H}_{k+1}$ is the Jacobian matrix of the measurement function. As an example of the application of the EKF to the OD of ESMO, a three-day OD campaign using Raisting as ground station, and a Ku-band transponder was simulated. Table 4 reports the errors which were used in the simulations.

Table 6, Figs. 16 and 17 report the results of the OD process and show the convergence history of the filtering process in number of filter iterations. Note that, the difference between the exact state and the estimated one at the end of the OD campaign fulfils the requirements derived in previous sections. These results confirm 


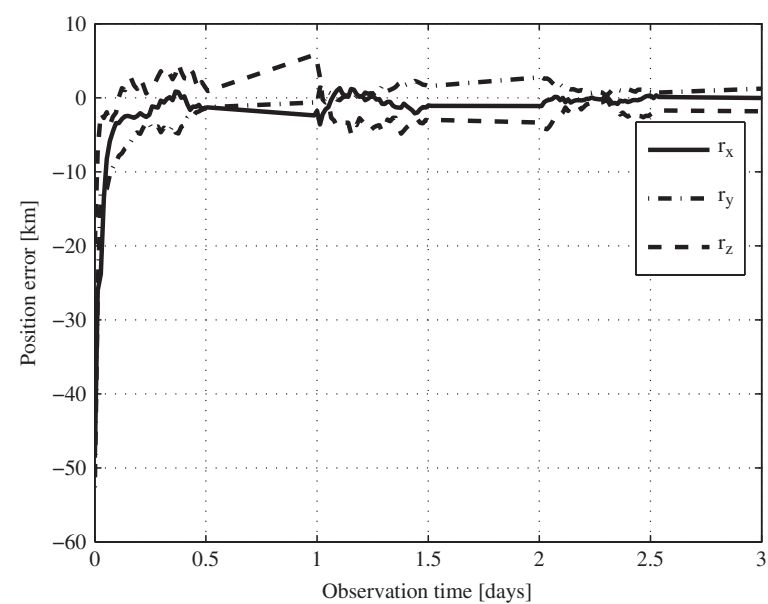

Fig. 16. Position error of the filtered measurements.

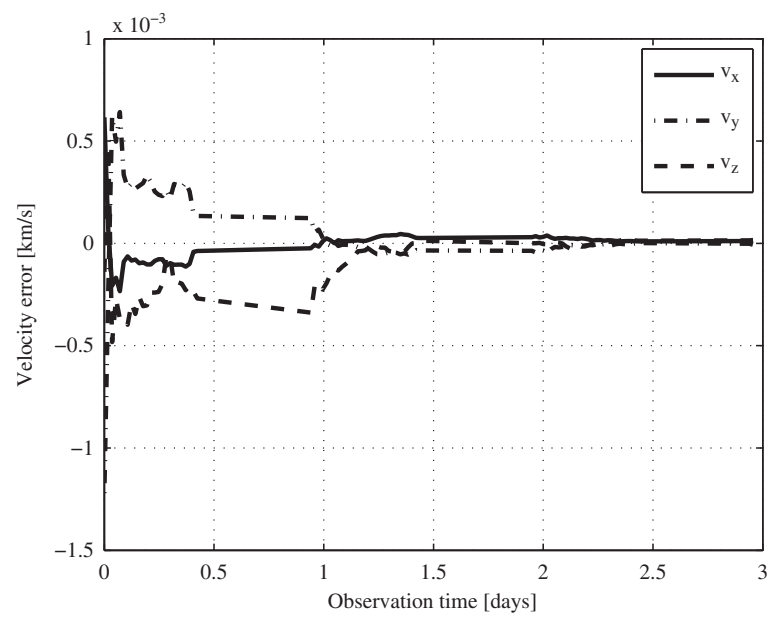

Fig. 17. Velocity error of the filtered measurements.

that the filter is able to handle the current measurement and process noise and correctly respond to the mission requirements.

\section{Navigation strategy}

Besides setting the upper limits on the measurements errors, the capture corridor provides the basis for the definition of a robust navigation strategy. The premise is to manoeuvre and maintain ESMO within the capture corridor at all times with enough margin to accommodate any orbit determination error. Intermingled along the transfer and in between the orbit determination segments are TCM's optimally timed to remain within the corridor till insertion. The goal of each TCM is that of minimising the deviation from the nominal trajectory at a certain point along the transfer. To ensure capture around the Moon, ESMO must be inside the capture corridor at all times. Therefore, after each orbit determination segment a TCM may, or may not, be required.
Orbit determination is assumed to occur over a three day period before each planned corrective action. This is to guarantee a good level of convergence of the filter. It is also assumed that the first orbit determination process occurs one week after the trans-lunar injection manoeuvre is performed.

Throughout the navigation analysis process, a number of sources of inherent error were considered. The error in the major $\Delta v$ manoeuvres at the trans-lunar injection burn is assumed to range between 0 and $10 \mathrm{~m} / \mathrm{s}$ in every direction. It was also assumed that each TCM in itself was affected by an error (due to misfiring of the thrusters) that must be accounted for. The analysis was conducted assuming a manoeuvre error of $1 \%, 5 \%, 10 \%$ of the nominal correction component in every direction. As before, a symmetric interval $[-\varepsilon \varepsilon]$ around each nominal component of the $\Delta v$ was considered and values were sampled,

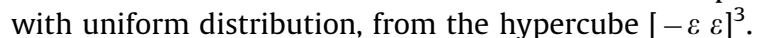

Following each TCM, the possible outcome of errors in both position and velocity of ESMO is measured at the next orbit determination point. In this sense, ESMO should be visible during all TCM's. The sum of all the TCM's will lead to an increase in the mission $\Delta v$ and propellant budget. Thus, the scheduling (time and date), direction and magnitude of the TCM's were optimised using the function fmincon of the MATLAB ${ }^{\mathrm{RM}}$ Optimisation Toolbox [22]. Two TCM's were allocated after each OD to correct the trajectory up to the next way point $t_{w p}$, i.e. the time along the trajectory where the spacecraft is expected to be within the corridor. Two way points were used in this analysis: one is the WSB point, i.e. when the spacecraft starts flying back towards the Moon, and the other is one week before lunar orbit injection. At the way point the nominal state of the spacecraft is $\mathbf{x}_{\text {nominal }}\left(t_{w p}\right)$ and the state provided by the implementation of the TCM's is $\mathbf{x}\left(t_{w p}\right)$. Each TCM is defined by its time of execution $t_{T C M}$ and the components of the velocity variation with respect to the local velocity vector. Note that a minimum delay of $12 \mathrm{~h}$ is inserted between the end of the OD and the first TCM, and also a minimum delay of $24 \mathrm{~h}$ between the first and second TCM, and a minimum delay of $12 \mathrm{~h}$ between the second TCM and the time at way point $t_{w p}$. These time spans are allocated in order to collect the telemetry data prior to manoeuvre planning and to upload the commands necessary to implement the correction manoeuvre. The function fmincon was then used to solve the following constrained optimisation problem:

$$
\begin{aligned}
& \min _{\mathbf{u} \in U} \Delta v_{T C M_{1}}+\Delta v_{T C M_{2}} \\
& \text { s.t. } \\
& \quad \mathbf{x}\left(t_{w p}\right)-\mathbf{x}_{\text {nominal }}\left(t_{w p}\right)=0
\end{aligned}
$$

with solution vector $\mathbf{u}=\left[t_{T C M 1} \Delta v_{T C M_{1 / x}} \Delta v_{T C M_{1 / y}} \Delta v_{T C M_{1 / z}}\right.$ $\left.t_{T C M_{2}} \quad \Delta v_{T C M_{2 / x}} \quad \Delta v_{T C M_{2 / y}} \quad \Delta v_{T C M_{2 / z}}\right]^{T} \in U$. The solution space $U$ is defined by the following lower and upper boundaries: $\mathbf{u}_{l}=\left[t_{\text {end OD }}+12 \mathrm{~h}-0.1 \mathrm{~km} / \mathrm{s}-0.1 \mathrm{~km} / \mathrm{s}-0.1 \mathrm{~km} / \mathrm{s}\left(t_{\text {endOD }}+t_{w p}\right) /\right.$ $2+12 \mathrm{hs}-0.1 \mathrm{~km} / \mathrm{s}-0.1 \mathrm{~km} / \mathrm{s}-0.1 \mathrm{~km} / \mathrm{s}]$ and $\mathbf{u}_{u}=\left[\left(t_{\text {endoD }}+\right.\right.$ $\left.t_{w p}\right) / 2-12$ h $0.1 \mathrm{~km} / \mathrm{s} 0.1 \mathrm{~km} / \mathrm{s} 0.1 \mathrm{~km} / \mathrm{s} t_{w p}-12 \mathrm{~h} 0.1 \mathrm{~km} / \mathrm{s}$ $0.1 \mathrm{~km} / \mathrm{s} 0.1 \mathrm{~km} / \mathrm{s}$ ], where $t_{\text {end } \mathrm{OD}}$ is the end time of the last OD campaign preceding the allocation of the TCM's. In order 
to collect sufficient data on the probability of success of the OD and navigation process, 100 repeated simulations were run for each scenario. Each scenario is defined by different ground station characteristics, number of allocated TCM's and error magnitude for each manoeuvre. In accordance to the current mission requirements all measurements for orbit determination will be gathered from the Raisting tracking stations. The number of OD campaigns is set a priori and their distribution along the trajectory is such that they provide good access to the ground station and good navigation capabilities during critical flight phases. The first OD for each leg is performed within 3 days after the TLI and the WSB manoeuvres. Prompt OD and Navigation are able to reduce the effects and errors such manoeuvres can introduce into the trajectory. Note that although two TCMs are normally allocated after each OD campaign, if the required correction is negligible the TCM is not performed. Furthermore, because the time of the TCMs is optimised and one OD campaign follows each executed TCM within 10 days, also the time of the OD is defined to optimally accommodate the required TCMs.

Fig. 18 illustrates a possible navigation strategy. This includes eight TCM's and six orbit determination campaigns, each one lasting for three days. After the initial translunar injection burn, a first orbit determination campaign is performed. Using the outcome of the orbit determination process, two TCM's are allocated taking as way point the WSB point. However, only one of them is performed before a second orbit determination process occurs. After the second orbit determination campaign, another two TCM's are planned, and so on and so forth till the first way point is reached. On the way back to the Moon, the same OD and Navigation process is repeated using the same method until ESMO reaches one week prior to lunar insertion. An example is presented in Table 7. The table reports the beginning and end of each orbit determination campaign and the date and magnitude of the allocated TCM.

Note that the TCM4 includes the WSB manoeuvre, the magnitude of which in the nominal solution, is $49.5 \mathrm{~m} / \mathrm{s}$, therefore the total navigation budget is the sum of all the

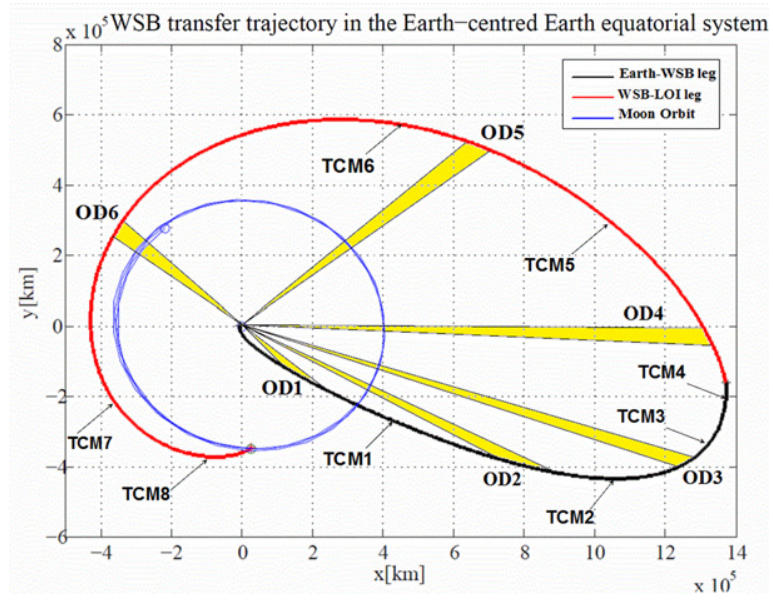

Fig. 18. Example of a possible navigation strategy.
Table 7

Example of Orbit Determination (OD) and TCM allocation for a 1\% error in the $\Delta v$ components of each manoeuvre.

\begin{tabular}{|c|c|}
\hline GTO-WSB Leg & \\
\hline \multirow[t]{2}{*}{ OD 1} & Start $19 / 03 / 2011$ \\
\hline & End $22 / 03 / 2011$ \\
\hline TCM 1 & $22 / 03 / 2011, \Delta V=6.4 \mathrm{~m} / \mathrm{s}$ \\
\hline \multirow[t]{2}{*}{ OD 2} & Start $02 / 04 / 2011$ \\
\hline & End $05 / 04 / 2011$ \\
\hline TCM 2 & $06 / 04 / 2011, \Delta V=0.1 \mathrm{~m} / \mathrm{s}$ \\
\hline \multirow[t]{2}{*}{ OD 3} & Start $17 / 04 / 2011$ \\
\hline & End $20 / 04 / 2011$ \\
\hline TCM 3 & $21 / 04 / 2011, \Delta V=0.15 \mathrm{~m} / \mathrm{s}$ \\
\hline TCM 4 & $26 / 04 / 2011, \Delta V=50.2 \mathrm{~m} / \mathrm{s}$ \\
\hline \multicolumn{2}{|l|}{ Moon-WSB Leg } \\
\hline \multirow[t]{2}{*}{ OD 4} & Start 04/05/2011 \\
\hline & End $07 / 05 / 2011$ \\
\hline TCM 5 & $07 / 05 / 2011, \Delta V=2.8 \mathrm{~m} / \mathrm{s}$ \\
\hline \multirow[t]{2}{*}{ OD 5} & Start $17 / 05 / 2011$ \\
\hline & End $20 / 05 / 2011$ \\
\hline TCM 6 & $20 / 05 / 2011, \Delta V=0.28 \mathrm{~m} / \mathrm{s}$ \\
\hline \multirow[t]{2}{*}{ OD 6} & Start 30/05/2011 \\
\hline & End $02 / 06 / 2011$ \\
\hline TCM 7 & $02 / 06 / 2011, \Delta V=0.11 \mathrm{~m} / \mathrm{s}$ \\
\hline TCM 8 & $08 / 06 / 2011, \Delta V=2.8 \mathrm{~m} / \mathrm{s}$ \\
\hline Total TCM $\Delta V(\mathrm{~m} / \mathrm{s})$ & 62.4 \\
\hline Additional $\Delta V(\mathrm{~m} / \mathrm{s})$ & 11.9 \\
\hline wrt nominal solution & \\
\hline
\end{tabular}

Table 8

Navigation statistics for different levels of accuracy in the execution of the manoeuvres, and assuming Ku-band data link for orbit determination.

\begin{tabular}{llll}
\hline Error on $\Delta v$ & $1 \%$ & $5 \%$ & $10 \%$ \\
\hline $\begin{array}{l}\text { Position }(\mathrm{km}) \\
\quad \text { Mean }\end{array}$ & 7.4 & 13.3 & 39.4 \\
$\quad$ Std & 4.2 & 7.9 & 28.2 \\
$\quad$ Velocity $(\mathrm{km} / \mathrm{s})$ & & & \\
$\quad$ Mean & $2.26 \times 10^{-5}$ & $9.12 \times 10^{-5}$ & $2.60 \times 10^{-4}$ \\
$\quad$ Std & $1.40 \times 10^{-5}$ & $6.65 \times 10^{-5}$ & $2.13 \times 10^{-4}$ \\
$\quad$ & & \\
Extra $\Delta V(\mathrm{~m} / \mathrm{s})$ & & 14.1 & 21.2 \\
$\quad$ Mean & 9.0 & 4.5 & 6.0 \\
$\quad$ Std & 3.8 & 0 & 0 \\
$\quad$ Solutions outside & 0 & & \\
$\quad 1$ week corridor & & &
\end{tabular}

TCM's minus the nominal WSB manoeuvre. Table 8 shows the mean and variance over 100 runs of the position and velocity achieved at the second way point and the corresponding navigation budget for different errors in the components of all the $\Delta v$ manoeuvres (including TCM's).

The last row of Table 8 reports the number of sampled transfers that are outside the corridor at one week from lunar orbit insertion. As expected a higher error in the execution of the manoeuvre (for example due to the poor control of the thrust vector) reduces the controllability of the system and therefore the final error in position and velocity is higher than in the case of a well controllable 
system. Nonetheless for the selected baseline the maximum navigation budget results to be slightly above $20 \mathrm{~m} / \mathrm{s}$ with no solutions left outside the capture corridor.

Figs. 19 and 20 show an example of final velocity at one week before LOI obtained implementing the proposed OD/TCM process for both a $5 \%$ error in the $\Delta v$ manoeuvres. The dots represent the back propagation from LOI, i.e. the corridor, while the stars the 100 simulated transfers.

In order to assess the worst case scenario for the orbit determination process it was assumed that Raisting was using a link in S-band thus having a higher beam width of about 50 arcsec. The navigation strategy still consists of six orbit determination campaigns (three days each) and eight TCM's executed throughout the transfer.

As shown in Table 9, despite the reduced performance, the additional required $\Delta v$ is limited to a maximum of $28 \mathrm{~m} / \mathrm{s}$ on average $(40 \mathrm{~m} / \mathrm{s}$ including a $3 \sigma$ standard deviation). In this case the effect of errors on both OD and corrections manoeuvre is magnified, increasing the navigation budget by $48 \%$ for a $10 \%$ error in the $\Delta v$ components.

\section{Discussion}

The analysis in this paper has shown the potentiality of an OD approach based on an extended Kalman filter, and the associated navigation strategy, for a low cost mission like ESMO. However, before an actual operational implementation of both the OD and navigation approaches in this paper, a number of extensions are required to incorporate a higher fidelity dynamic, ground segment and space segment models. This extension includes the effect of solar pressure during coasting arcs, the accurate modelling of all manoeuvres considering the actual thrust profile (which is dependent on both the propulsion and the attitude subsystems) and higher fidelity model of the measurements. In this respect some recent analyses [23] have shown the effect of the error in the pointing angle

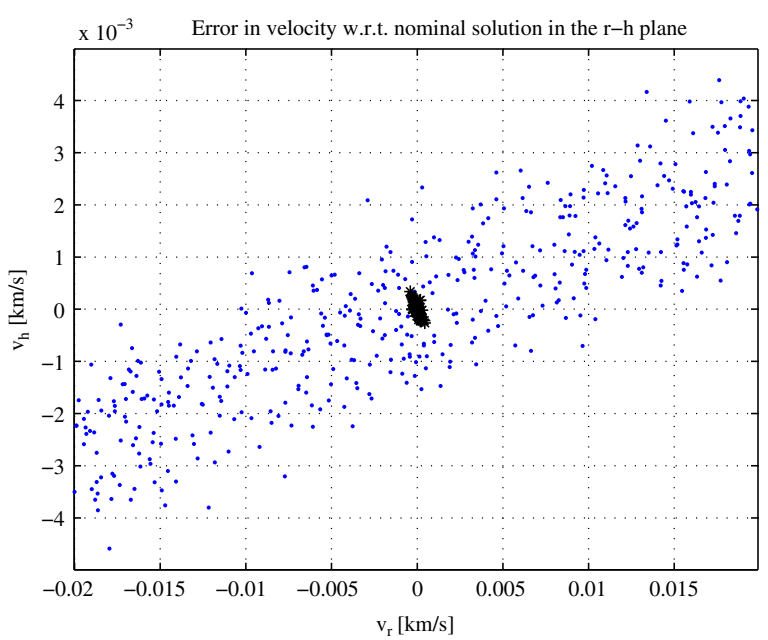

Fig. 20. Error in position in the $r-h$ plane: corridor (blue dot), corrected trajectory (black asterisk). (For interpretation of the references to colour in this figure legend, the reader is referred to the web version of this article.)

\section{Table 9}

Transfer statistics for different levels of thrust and ADCS accuracy, using S-band for orbit determination.

\begin{tabular}{llll}
\hline Error on $\Delta v$ & $1 \%$ & $5 \%$ & $10 \%$ \\
\hline $\begin{array}{l}\text { Position }(\mathrm{km}) \\
\quad \text { Mean }\end{array}$ & 34.9 & 36.3 & 54.1 \\
$\quad \sigma$ & 19.2 & 19.9 & 26.7 \\
$\quad$ & & \\
$\quad$ Velocity $(\mathrm{km} / \mathrm{s})$ & & & $2.56 \times 10^{-4}$ \\
$\quad$ Mean & $9.92 \times 10^{-5}$ & $1.23 \times 10^{-4}$ & $2.72 \times 10^{-4}$ \\
$\sigma$ & $6.86 \times 10^{-5}$ & $7.58 \times 10^{-5}$ & \\
Extra $\Delta V(\mathrm{~m} / \mathrm{s})$ & & & 28.0 \\
$\quad$ mean & 10.5 & 14.1 & 12.0 \\
$\quad \sigma$ & 3.7 & 4.6 & 0 \\
$\quad$ Solutions outside & 0 & 0 & \\
$\quad 1$ week corridor & & & \\
\hline
\end{tabular}

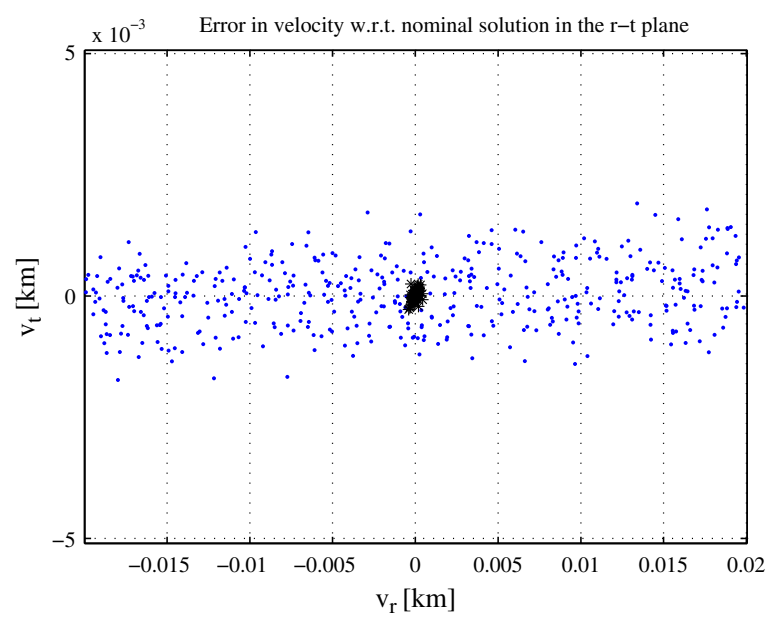

Fig. 19. Error in position in the $r$ - $t$ plane: corridor (blue dot), corrected trajectory (black asterisk). (For interpretation of the references to colour in this figure legend, the reader is referred to the web version of this article.) 
measurements the pointing angles in comparison to an OD strategy based only on range and range-rate measurements. Although, the corridor-based navigation strategy is robust against inaccuracies in measurements and TCM execution, the quality of the measurements might lead to a redefinition of the OD strategy or a relaxation of the requirements on the final lunar orbit.

Furthermore, an optimal allocation of the last OD campaign and TCM has shown that in a number of cases a correction at less than one week from orbit insertion might be appropriate. This suggests that once a baseline transfer is available the whole OD and navigation strategies will need to be accurately tailored on that specific baseline. Because one of the main requirements for ESMO is to have a transfer solution for every day of the launch window, the OD and navigation strategies will need to be defined (or redefined) with very short notice to accommodate the availability of launch opportunities.

\section{Concluding remarks}

This paper presented a first analysis of the orbit determination requirements and possible navigation strategies for ESMO. The proposed corridor-targeting approach yields good results at a relatively low $\Delta v$ cost. This is coupled with mild orbit determination accuracy. This approach therefore seems to be ideal for small spacecraft missions that are constrained with a low mission $\Delta v$. However, in the assessment of the current navigation strategy, still a number of sources of noise need to be considered, in particular the accuracy of the ground station tracking system related to the ionospheric effects and process noise due to unmodelled components. The final design and construction of the ground station will allow using actual measurement data, in order to validate and consolidate the analysis. The data suggest that less accurate tracking systems and higher error in the TCM's could lead to velocity poorly controllable system and a difficult targeting of the corridor. On the other hand the current targeting strategy considers a conservative $1 \%$ error and the exact satisfaction of the terminal constraint when planning the TCM's. Integrating attitude dynamics and propulsion systems into the flight dynamics will allow precisely simulating the manoeuvres and their impacts on the navigation. A more flexible strategy is under investigation that aims at a weak temporary capture in case of major contingencies or underperformance of the engines.

An autonomous navigation experiment is currently under study in order to provide navigation and control during one week of autonomous operations. The idea is to use attitude sensors and payload camera, in order to perform on-board trajectory estimation and operate the spacecraft autonomously, thus improving the likelihood of the mission success. Furthermore, throughout this analysis the number of TCM's was not optimised. Further work will address this optimisation and a more tailored orbit determination process of ESMO.

\section{References}

[1] D. Lozier, K. Galal, D. Folta, M. Beckman, Lunar Prospector mission design and trajectory support, Adv. Astronaut. Sci. 100 (1998) 297-312.

[2] H. Mizutani, A. Fujimura, S. Tanaka, H. Shiraishi, T. Nakajima, Lunar-A mission: goals and status, Adv. Space Res. 31 (11) (2003) 2315-2321.

[3] J.N. Goswami, M. Annadurai, Chandrayaan-1: India's first planetary science mission to the Moon, Curr. Sci. 96 (4) (2009).

[4] R. Walker, M. Cross, The European Student Moon Orbiter (ESMO): lunar mission for education, outreach and science, Acta Astronaut. 66 (7-8) (2010) 1177-1188.

[5] ESA, ESA Education-ESMO Mission. Available online at: 〈http://www. esa.int/esaMI/Education/SEMLOMPR4CF_0.html >, 2010 (last assessed August 2010)

[6] D.W. Dunham, R.W. Farquhar, Trajectory design for a lunar mapping and Near-Earth-Asteroid Flyby Mission, AAS 93-145, Spaceflight Mechanics 1993, Adv. Astronaut. Sci. (1993) 605-624.

[7] D. Haros, J. Schoenmaekers, Post-launch optimisation of the Smart 1 low-thrust trajectory to the Moon, in: Proceedings of the 18th International Symposium on Spaceflight Dynamics, Munich, Germany, 15-22 October, 2004.

[8] R.W. Farquhar, D.W. Dunham, Y. Gao, J.V. McAdams, Utilization of libration points for human exploration in the Sun-Earth-Moon system and beyond, in: Proceedings of the 54th International Astronautical Congress of the International Astronautical Federation, Bremen, Germany, September-October, 2003, Acta Astronaut. 55 (2004) 687-700

[9] E.A. Belbruno, J.K. Miller, Sun-perturbed Earth-to-Moon transfers with ballistic capture, J. Guidance Control Dyn. 16 (1993).

[10] ESA, ESMO_PhaseA_MIAS_A1_Mission_Analysis_Report_20090309 2009.

[11] E. Brandon, B.N. Vincent, B. Olberts, D. Novak, ESMO Phase B1 Mission analysis: targeting options for lunar WSB transfers along with a multi-burn injection strategy, IAC-09.E2.3.5, in: Proceedings of the 60th International Astronautical Congress, 12-16 October 2009.

[12] P. Dysli, Analytical Ephemeris for Planets, 1977

[13] P.K. Seidelmann, et al., Report of the IAU/IAG Working Group on cartographic coordinates and rotational elements: 2006, Celestia Mech. Dyn. Astron. 98 (3) (2007) 155-180

[14] E.R. Dam, B.G.M. van Husslage, D. den Hertog, J.B.M. Melissen, Maximin Latin hypercube designs in two dimensions, Oper. Res. 55 (2007) 158-169.

[15] Analytical Graphics, Inc., 220 Valley Creek Blvd., Exton, PA 19341, USA, Phone: 1.610.981.8000, info@agi.com, 〈http://www.agi.com〉.

[16] A.S. Konopliv, S.W. Asmar, E. Carranza, W.L. Sjogren, D.N. Yuan, Recent gravity models as a result of the Lunar Prospector mission, Icarus 150 (1) (2001) 1-18.

[17] ESA, European Student Moon Orbiter System Requirements Document-Phase B1, Issue 3, REF: ESMO_PhaseB1_SRD_ 20090309, 2009.

[18] R.H. Battin, G.M. Levine, Applications of Kalman filtering techniques to the Apollo program, in: C.T. Leondes (Ed.), Theory and Applications of Kalman filtering, 1970, pp. 335-361.

[19] D.A. Vallado, W.D. McClain, Fundamentals of Astrodynamics and Applications, 2nd edition, , 2004 (Space Technology Library).

[20] O. Montenbruck, E. Gill, Satellite Orbits: Models, Methods and Applications, Springer, 2000.

[21] J.L. Crassidis, J.L. Junkins, Optimal Estimation of Dynamic Systems, in: S. Haykin (Ed.), 2001.

[22] 〈http://www.mathworks.com/help/toolbox/optim/ug/fmincon.html 〉, MathWorks $^{\mathrm{TM}}$.

[23] M. Vetrisano, M. Vasile, Navigating to the Moon along low-energy transfers: the case of the European Student Moon Orbiter, in: Proceedings of the 62nd International Astronautical Congress, Paper ID: IAC-11.A 3.2A.5, Cape Town, SA, October 2011. 